\title{
Real-time computation of transcranial magnetic stimulation electric fields using self- supervised deep learning
}

\author{
Hongming Li a ${ }^{\text {, Zhi-De Deng }}{ }^{\text {, }}$, Desmond Oathes ${ }^{c}$, Yong Fan ${ }^{a,{ }^{*}}$ \\ a Center for Biomedical Image Computation and Analytics, Department of Radiology, Perelman School of \\ Medicine, University of Pennsylvania, Philadelphia, PA 19104, USA \\ ${ }^{b}$ Computational Neurostimulation Research Program, Noninvasive Neuromodulation Unit, Experimental \\ Therapeutics \& Pathophysiology Branch, National Institute of Mental Health, NIH, MD 20892, USA \\ c Center for Neuromodulation in Depression and Stress, Department of Psychiatry, Perelman School of Medicine, \\ University of Pennsylvania, Philadelphia, PA 19104, USA
}

${ }^{*}$ Correspondence: Yong.Fan@pennmedicine.upenn.edu

\section{Abstract}

Background: Electric fields (E-fields) induced by transcranial magnetic stimulation (TMS) can be modeled using partial differential equations (PDEs) with boundary conditions. However, existing numerical methods to solve PDEs for computing E-fields are usually computationally expensive. It often takes minutes to compute a high-resolution E-field using state-of-the-art finite-element methods (FEM).

Methods: We developed a self-supervised deep learning (DL) method to compute precise TMS E-fields in real-time. Given a head model and the primary E-field generated by TMS coils, a self-supervised DL model was built to generate a E-field by minimizing a loss function that measures how well the generated E-field fits the governing PDE and Neumann boundary condition. The DL model was trained in a selfsupervised manner, which does not require any external supervision. We evaluated the DL model using both a simulated sphere head model and realistic head models of 125 individuals and compared the accuracy and computational efficiency of the DL model with a state-of-the-art FEM.

Results: In realistic head models, the DL model obtained accurate E-fields with significantly smaller PDE residual and boundary condition residual than the FEM ( $p<0.002$, Wilcoxon signed-rank test). The DL model was computationally efficient, which took about 0.30 seconds on average to compute the E-field for one testing individual. The DL model built for the simulated sphere head model also obtained an accurate E-field whose difference from the analytical E-fields was 0.004 , more accurate than the solution obtained using the FEM.

Conclusions: We have developed a self-supervised DL model to directly learn a mapping from the magnetic vector potential of a TMS coil and a realistic head model to the TMS induced E-fields, facilitating real-time, precise TMS E-field modeling.

Keywords: Self-supervised learning, deep neural networks, electric field modeling, TMS 


\section{Introduction}

Transcranial magnetic stimulation (TMS) is a noninvasive brain stimulation method used in treating major depression and other neuropsychiatric disorders [1]. However, TMS treatment outcomes vary greatly across patients [2-12]. One primary source of such variability is that TMS suffers from targeting inaccuracies $[13,14]$. Recent studies have demonstrated that optimizing TMS stimulation parameters, such as location and orientation of the TMS coil, might improve TMS targeting and focality for individual subjects [15-17], and optimizing TMS coil placement based on individual functional neuroanatomy could potentially increase effect sizes for both basic and clinical studies [2-8].

Modeling of electric-fields (E-fields) is now the most widely used method to characterize the localization and spread of electrical current in the brain induced by TMS [18-23]. A variety of numerical computational methods have been developed to compute E-fields in conjunction with realistic head models by iteratively solving PDEs governing the E-field induced by TMS coils, including finite element methods (FEMs), boundary element methods (BEMs), and finite-difference methods (FDMs) [21, 24-27] [28]. The convergence of these numerical methods to the actual solution is sensitive to density of the head mesh, the polynomial approximation order, and error tolerance, and their computational cost is proportional to the modeling accuracy [29]. Therefore, compromise is often necessary in real applications. It takes minutes for state-of-the-art E-field modeling methods to compute a high-resolution E-field [21, 24-27].

The high computational cost of E-field modeling also makes it time-consuming and costly to optimize TMS stimulation parameters since a large number of E-fields have to be explored to identify an optimal solution [15-17]. To benefit clinical practice using these sophisticated tools, the computational cost of E-field modeling has to be reduced substantially without compromising accuracy. Faster E-field modeling is achievable by using a dipole-based magnetic stimulation profile approach that has to compute a magnetic stimulation profile for each individual subject with several hours of CPU time [30] or to compute the E-field only on sparse points or the mean of the E-field in a region of interest (ROI) by leveraging the reciprocity principle [17, 31]. Recent studies have demonstrated that superfast highresolution E-field modeling can be achieved using deep neural networks (DNNs) [32, 33]. Particularly, the magnitude of E-fields was estimated based on individualized MRI head scans and TMS coil positions by DNNs [32], and 3D vector E-fields were predicted using deep DNNs by integrating both individualized neuroanatomy (scalar-valued tissue conductivity or anisotropic conductivity tensors) and primary E-fields generated by TMS coils as the input [33]. Though promising results have been obtained, the existing deep learning $(D L)$ based models were driven and optimized in the supervised learning setting. To train the DNNs, E-fields estimated by conventional numerical methods, such as FEM, are used to generate training data. Therefore, their accuracy would be bounded by the conventional numerical methods used 
to generate the training data.

Inspired by self-supervised deep learning methods [34-45] and the pioneer deep learning based E-field computation methods [32, 33], we develop a novel self-supervised deep learning based TMS Efield modeling method to obtain precise high-resolution E-fields in real-time. Specially, given a head model and the primary E-field generated by TMS coil, a DL model is built to generate the electric scalar potential by minimizing a loss function that measures how well the generated electric scalar potential fits the governing PDE and Neumann boundary condition, from which the E-field can be derived directly. In contrast to the conventional numerical methods that solve the PDEs iteratively, the DL model is built to learn the solution to the PDE directly. In contrast to the existing supervised DL methods, our DL model is trained in a self-supervised manner by minimizing the residuals of the governing PDE and boundary condition as a loss function, which does not require any external supervision. The trained DL model could be applied to new subjects and predict their E-fields by one forward-pass computation. We have validated the proposed DL model using both simulated sphere head model and realistic head models, and experimental results have demonstrated that it can obtain improved accuracy and computational efficiency compared with a state-of-the-art FEM implemented in SimNIBS v3.1.

\section{Methods}

We develop a self-supervised DL model to compute TMS E-fields by directly learning a mapping from the magnetic vector potential of a TMS coil and a realistic head model to the TMS induced E-field so that high-resolution TMS E-fields will be a good estimate of the solution to the governing PDE of TMS E-fields and be computed by one feedforward computation rapidly.

\section{TMS E-fields modeling}

Given a head model consisting of head tissue compartments with different conductivities, the $\mathrm{E}$-field $E$ in the head induced by a TMS coil can be computed by solving a PDE [20, 22, 23]. Based on quasi-static approximation, the E-field, $E=-\nabla \phi-\frac{\partial A}{\partial t}$, can be computed by solving

$$
\nabla \cdot(\sigma \nabla \phi)+\nabla \cdot\left(\sigma \frac{\partial A}{\partial t}\right)=0
$$

with the Neumann boundary condition

$$
\vec{n} \cdot\left(\sigma \nabla \phi+\sigma \frac{\partial \mathrm{A}}{\partial \mathrm{t}}\right)=0
$$

where $\sigma$ is the tissue conductivity, $A$ is the magnetic vector potential of the TMS coil, $\phi$ is the electric

scalar potential, and $\vec{n}$ is the normal vector to the tissue surface. Particularly, the primary E-Field $-\frac{\partial A}{\partial t}$ depends only on the TMS coil characteristics $[18,46]$ and the secondary field $-\nabla \phi$ is caused by surface 
charges in the conducting medium characterized by the head model.

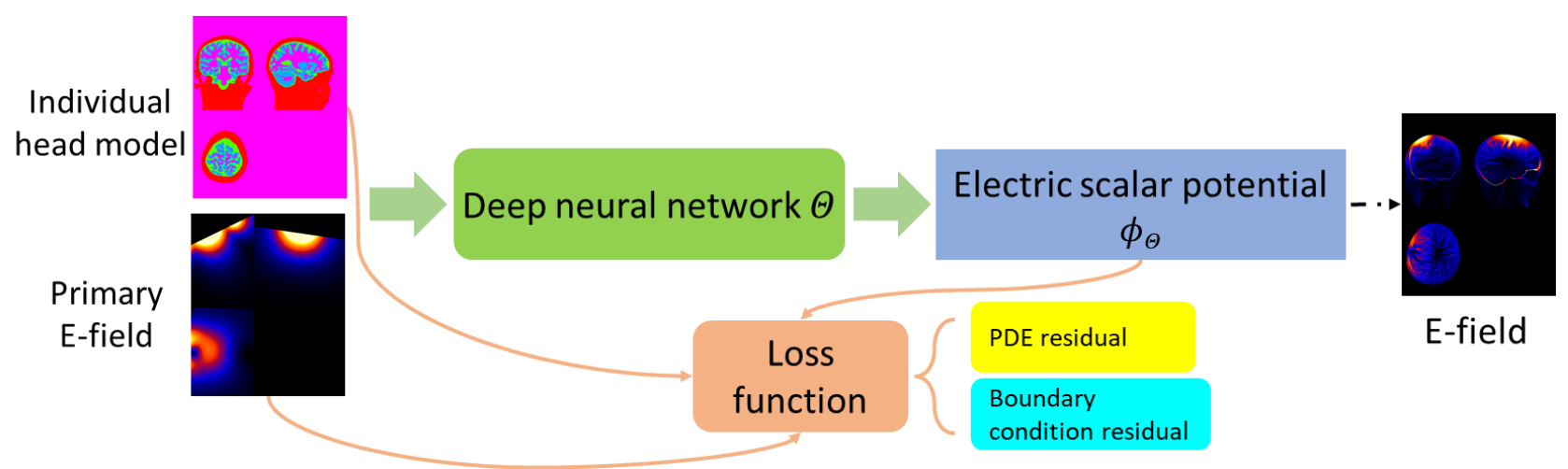

Fig.1. A self-supervised deep learning model for computing TMS E-field. A deep neural network is applied to learn a mapping from individual head model and TMS induced primary E-field to the TMS E-field, and the network is optimized by a loss function consisting of PDE residual and boundary condition residual in a self-supervised learning manner.

\section{Computing TMS E-fields using deep neural networks}

In contrast to the prevailing FEM/BEM methods adopted in E-field modeling studies [15-17, 21, 24-26, 31] and the existing DL method that learns a mapping from individual MRI head scans/anatomy to Efields in a supervised learning framework [32, 33], our DL model is built to solve Eq. (1) with the boundary condition of Eq. (2) in a self-supervised fashion as illustrated in Fig. 1. Given a head model and TMS induced primary E-field as input, a deep neural network with parameters $\Theta$ is built to estimate $\phi$ by minimizing a loss function $L$ that measures how well the generated $\phi$ fits the PDE and Neumann boundary condition. The loss function $L$ is specified as:

$$
\begin{gathered}
\min _{\Theta} L\left(\Theta ;\left\{v^{i}\right\}_{i=1}^{N}\right)=\frac{1}{N} \sum_{i=1}^{N}\left[G\left(\phi_{\Theta}\left(v^{i}\right)\right)+\lambda B\left(\phi_{\Theta}\left(v^{i}\right)\right)\right], \\
G\left(\phi_{\Theta}\right)=\left(\nabla \cdot\left(\sigma \nabla \phi_{\Theta}\right)+\nabla \cdot\left(\sigma \frac{\partial A}{\partial t}\right)\right)^{2} \\
B\left(\phi_{\Theta}\right)=\left(\vec{n} \cdot\left(\sigma \nabla \phi_{\Theta}+\sigma \frac{\partial \mathrm{A}}{\partial \mathrm{t}}\right)\right)^{2}
\end{gathered}
$$

where $G\left(\phi_{\Theta}\right)$ and $B\left(\phi_{\Theta}\right)$ are residuals of the governing PDE and boundary condition respectively, $\phi_{\Theta}$ is the electric scalar potential computed by the deep neural network, $\left\{v^{i}\right\}_{i=1}^{N}$ refers to all the spatial locations (voxels) within the head model, and $\lambda$ is a trade-off hyper-parameter for balance between $G\left(\phi_{\Theta}\right)$ and $B\left(\phi_{\Theta}\right)$. For clarity, $v^{i}$ is omitted in Eq. (4) and Eq. (5). 
To facilitate robust training of the deep neural network, a mixed variable formulation [42] with auxiliary variable $\tau_{\Theta}=-\sigma \nabla \phi_{\Theta}$ is adopted and the auxiliary variable is estimated by the deep neural network as an auxiliary output (Fig. 2). The overall loss function $L$ becomes

$$
\begin{gathered}
\min _{\Theta} L\left(\Theta ;\left\{v^{i}\right\}_{i=1}^{N}\right)=\frac{1}{N} \sum_{i=1}^{N}\left[G_{\text {mix }}\left(\phi_{\Theta}\left(v^{i}\right), \tau_{\Theta}\left(v^{i}\right)\right)+\lambda B_{\text {mix }}\left(\phi_{\Theta}\left(v^{i}\right), \tau_{\Theta}\left(v^{i}\right)\right)\right], \\
G_{\text {mix }}\left(\phi_{\Theta}, \tau_{\Theta}\right)=\left(\tau_{\Theta}+\sigma \nabla \phi_{\Theta}\right)^{2}+\left(-\nabla \cdot \tau_{\Theta}+\nabla \cdot\left(\sigma \frac{\partial \mathrm{A}}{\partial \mathrm{t}}\right)\right)^{2}, \\
B_{\text {mix }}\left(\phi_{\Theta}, \tau_{\Theta}\right)=\left(\vec{n} \cdot\left(\sigma \nabla \phi_{\Theta}+\sigma \frac{\partial \mathrm{A}}{\partial \mathrm{t}}\right)\right)^{2}+\left(\vec{n} \cdot\left(-\tau_{\Theta}+\sigma \frac{\partial \mathrm{A}}{\partial \mathrm{t}}\right)\right)^{2},
\end{gathered}
$$

where $G_{\text {mix }}\left(\phi_{\Theta}, \tau_{\Theta}\right)$ and $B_{\text {mix }}\left(\phi_{\Theta}, \tau_{\Theta}\right)$ are residuals of the governing PDE and boundary condition with $\tau_{\Theta}$ incorporated. Given the input training data, the deep neural network is trained to optimize the loss function of Eq. (6) in a self-supervised manner. Once the deep neural network is optimized, it could be applied to new subjects and predict the electric potential $\phi$ by one forward-pass computation, from which the Efields could be computed directly.

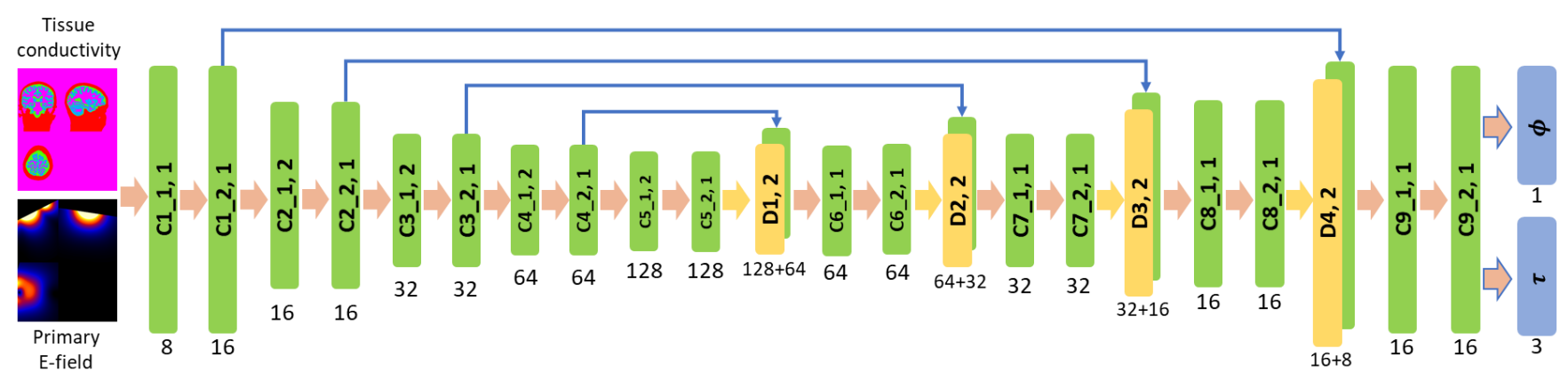

Fig.2. Deep convolutional neural network with an Encoder-Decoder architecture applied to learn TMS E-field. The numbers underneath convolutional $\left(\mathrm{C} 1 \_1, \mathrm{C} 1{ }_{2}, \ldots, \mathrm{C}\right.$ _2) and deconvolutional (D1, D2, D3, and D4) layers indicate their corresponding numbers of kernels, with a stride of 1 or 2 for downsampling or upsampling. The kernel size in all layers is set to $3 \times 3 \times 3$.

\section{Network architecture for computing TMS E-fields}

The overall architecture of our deep neural network for computing TMS E-fields is illustrated in Fig. 2. The deep neural network's backbone is a U-Net with an Encoder-Decoder architecture [47]. The network's input consists of a 3D individual head model (tissue conductivity map) and a 3D subject-specific primary E-field $\left(-\frac{\partial A}{\partial t}\right)$, and its output is the estimated electric scalar potential $\phi_{\Theta}(3 \mathrm{D}$ volume with the same spatial dimension as the head model) and the auxiliary variable $\tau_{\Theta}$ (three $3 \mathrm{D}$ volumes with the same spatial dimension as the head model). The total E-field will be estimated as $E=-\nabla \phi_{\Theta}-\frac{\partial A}{\partial t}$. 
Particularly, the encoder path consists of ten convolutional layers with 8 to 128 filters and a stride of 1 or 2 for downsampling, the decoder path consists of four deconvolutional layers with 128, 64, 32, and 16 filters and a stride of 2 for upsampling, each of which is followed by two additional convolutional layers with $64,32,16$, and 16 filters and a stride of 1 . Two output convolutional layers with 1 and 3 filters are used to predict the electric scalar potential $\phi_{\Theta}$ and the auxiliary variable $\tau_{\Theta}$. Leaky ReLU [48] activation function is used for all the convolutional and deconvolutional layers, except those two output layers. The kernel size in all layers is set to $3 \times 3 \times 3$.

\section{Experimental results}

\section{Data preparation}

We have evaluated the proposed deep learning method using both simulated sphere head model and realistic head models from real MRI scans.

For the simulated data, a 3D sphere head model with a radius of $95 \mathrm{~mm}$ and isotropic resolution of $1 \mathrm{~mm}$ was generated. Its origin coordinate was set to [0,0,0] and its conductivity set to $1 \mathrm{~s} / \mathrm{m}$ homogeneously. The excitation was given by a point magnetic dipole located outside of the sphere. The dipole's location was set to $[0,0,300]$ and its moment set to $[0,0,1]$. The TMS induced E-field of this sphere head model can be calculated analytically [49], facilitating the direct evaluation of numerical accuracy of methods under comparison.

For the realistic head models, we adopted a local cohort of 125 healthy adult subjects with highresolution multi-echo T1-weighted MPR images (TR=2400 ms, $\mathrm{Tl}=1060 \mathrm{~ms}, \mathrm{TE}=2.24 \mathrm{~ms}, \mathrm{FA}=8^{\circ}$, $0.8 \times 0.8 \times 0.8 \mathrm{~mm}$ voxels, image size $=208 \times 300 \times 320, \mathrm{FOV}=256 \mathrm{~mm})$. Based on these MRI scans, we used SimNIBS v3.1 to generate anatomically accurate head models ('headreco' option with SPM/Computational Anatomy Toolbox for tissue segmentation) and compute primary E-fields induced by a Magstim 70mm Figure-of-Eight coil placed at varied locations with different orientations [50]. The tissue conductivity map was generated by substituting the head tissue label values with their corresponding conductivity values. We adopted the SimNIBS conductivity values, i.e., 0.126, 0.275, $1.654,0.01,0.465$, and $0.5 \mathrm{~S} / \mathrm{m}$ for whiter matter, gray matter, CSF, bone, scalp, and eyes respectively.

\section{Experiment settings and implementation}

To obtain the E-field of the simulated sphere head model using our proposed deep learning model, the conductivity map of the sphere and the primary E-field induced by the dipole was fed into the network as illustrated in Fig. 2, and the network was trained and optimized with respect to the loss function of Eq. (6) until convergence. 
For the evaluation on the realistic head models, we randomly selected 100 subjects as training subjects and the remaining 25 as testing subjects. To evaluate the accuracy and robustness of our deep learning model with respect to different TMS coil positions and directions, three different experiment settings were adopted.

Setting 1. The TMS coil was placed at a location within the motor cortex (center='C1' and pos_ydir='CP1' as defined in the EEG10-10 system) for each subject to generate a primary E-field using SimNIBS. There were 100 pairs of subject specific tissue conductivity maps and primary E-fields in total used for training the deep learning model and 25 pairs for testing its accuracy under this setting, which served as a proof-of-concept validation of our deep learning model on realistic head models.

Setting 2. The TMS coil was placed at the same location within the motor cortex (center='C1') but with different coil directions for training and testing the deep learning model. Particularly, primary E-fields of each training subject were generated with different coil directions (seen directions), including 'CP1', 'Cz', 'FC1', and 'C3', as training data. There were 400 pairs of subject specific tissue conductivity maps and primary E-fields in total for training the deep learning model. The optimized deep learning model was then evaluated on the testing subjects with the coil placed in directions different from those for generating the training data (unseen directions), including 'CPz', 'FCz', 'FC3', and 'CP3'. In total, there were 100 pairs of subject specific tissue conductivity maps and primary E-fields for testing. This setting was adopted to evaluate the generalization performance of the deep learning model with respect to varying coil directions.

Setting 3. The TMS coil was placed at different spatial locations within the left dorsolateral prefrontal cortex (DLPFC) to evaluate the robustness of the deep learning model with respect to changes of coil locations. Particularly, a target position was defined using the average mean Montreal Neurological Institute (MNI) coordinates $(x=-42, y=16, z=28$ ) [51], which was transformed to subject space using SIMNIBS to obtain a subject-specific target position. Then, multiple coil positions and directions were generated within a grid centered at the target position using the SIMNIBS function 'optimize_tms.get_opt_grid' with parameters of radius $=20$, resolution_pos $=10$, resolution_angle=90, angle_limits=[-180,180], yielding 36 (9 positions by 4 directions) pairs of tissue conductivity map and primary E-field for each subject. In total, there were 3600 pairs of subject specific tissue conductivity maps and primary E-fields for training the deep learning model and 900 pairs of subject specific tissue conductivity maps and primary E-fields for testing its accuracy.

For both the simulated and realistic head models, the input to our deep neural network consists of the tissue conductivity map (3D volume) and primary E-field (4D volume), the output of the network consists of the predicted scalar electric potential (3D volume) and the auxiliary variable $\tau_{\Theta}$ (4D volume). The deep neural network was optimized under each setting respectively based on the training data 
regarding the loss function of Eq. (6). The subject-specific head model, primary E-field, and the boundary normal vector image (4D volume) of the head model was generated using SimNIBS for each subject. For the realistic head models, a 3D/4D image patch focused on the TMS evoked area was cropped out of the original volume image to improve the computational efficiency. The spatial dimension of image patch was $112 \times 288 \times 80$ and $96 \times 272 \times 128$ for the data of TMS coil located at motor cortex and DLPFC, respectively. The channel size was 1 and 3 for scalar and vector image, respectively.

Our deep learning model was implemented using Tensorflow [52]. Adam optimizer [53] is adopted to optimize the network, the learning rate was set to $1 \times 10^{-4}$, the batch size was set to 1 , and the number of iterations is set to 10000 for the simulated sphere head model, 30000 for realistic head model setting 1, 60000 and 90000 for realistic head model setting 2 and 3 during training. One NVIDIA Tesla P100 GPU with $12 \mathrm{G}$ memory was used for training and testing. We set $\lambda=1$ empirically for the realistic head models and $\lambda=0.1$ for the simulated sphere head model in our experiments. Training losses of our deep learning models on the simulated sphere head model and realistic head models are shown in Fig. 3, demonstrating the optimized deep learning models reach convergence after training under the parameter settings used.

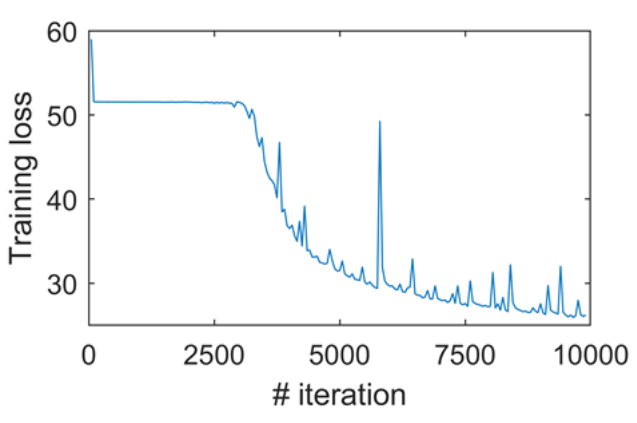

a

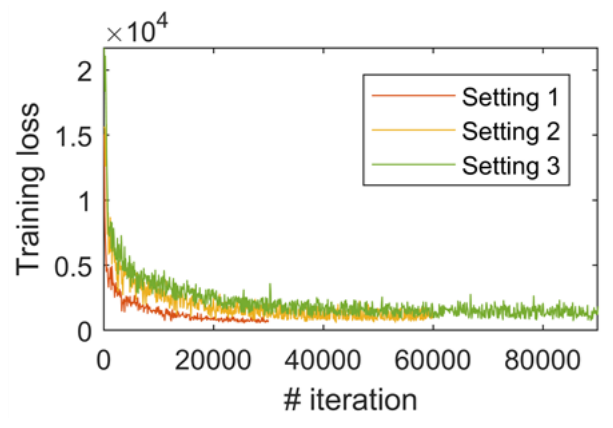

b

Fig.3. Training loss of the proposed deep learning model on simulated sphere head model (a) and realistic head models under different experiment settings (b).

\section{Evaluation and comparisons}

We compared our method in terms of both accuracy and computational efficiency with a state-of-the-art FEM implemented in SimNIBS v3.1. As no ground truth was available for the realistic head models, accuracy of E-fields was evaluated using the residuals of governing PDE and boundary condition as computed in Eq. (4) and Eq. (5). Wilcoxon signed-rank test was adopted to compare different E-field modeling methods in terms of their accuracy. For the simulated sphere head model, the E-field can be calculated analytically [49] to directly evaluate the numerical accuracy of methods under comparison. Particularly, the difference between a numerical solution $E_{\text {num }}$ and an analytically solution $E_{\text {ana }}$ was 
measured with a normalized root-mean-square error (NRMSE): $\frac{1}{N} \sum_{v=1}^{N} \frac{\left\|E_{\text {num }}(v)-E_{\text {ana }}(v)\right\|}{\left\|E_{\text {ana }}(v)\right\|}$, where $N$ is the number of voxels in the sphere head model.

\section{Results}

Results on simulated sphere head model. The magnitude of E-fields computed analytically, by the FEM, and by the proposed DL model are shown in Fig. 4. It can be observed that the E-field obtained by our DL model is visually similar to the analytical solution. The NRMSE between the DL based and analytical E-field was 0.004 , which is comparable to the E-field solution obtained by the FEM (NRMSE=0.008). In addition, the PDE residual and boundary condition residual of the DL based solution was 9.67 and 116.30 respectively, which were smaller than the PDE residual (26.17) and boundary condition residual (118.47) of the FEM based solution. This result demonstrated that the proposed DL model can indeed be optimized to learn the E-field that follows the physics law underlying the TMS stimulation.

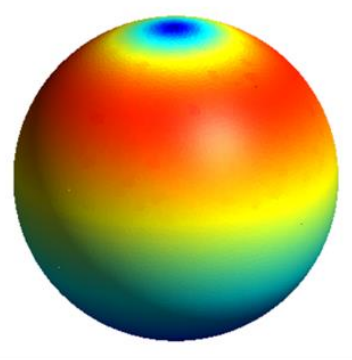

Analytical

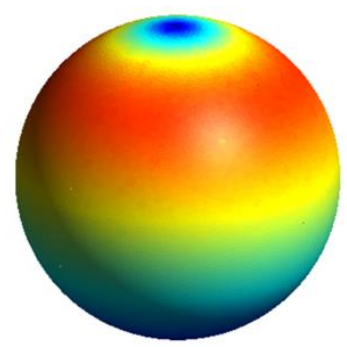

FEM

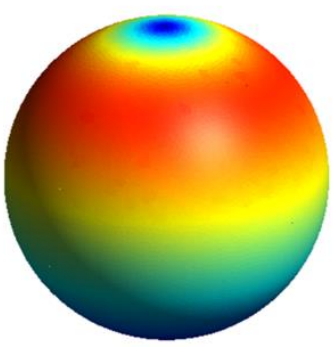

$\mathrm{DL}$

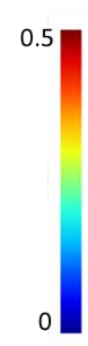

Fig.4. The magnitude of E-fields of the sphere model computed analytically, by FEM (with a NRMSE of 0.008 , a PDE residual of 26.17, and a boundary condition residual of 118.47), and by our DL method (with a NRMSE of 0.004 , a PDE residual of 9.67 , and a boundary condition residual of 116.30 ), respectively.

Results on realistic head model setting 1. The magnitude of E-fields of three randomly selected testing subjects computed by FEM and our proposed DL method are shown in Fig. 5a. The results obtained by our DL model have similar pattern to that obtained by FEM, and there are large overlaps between the regions with higher magnitude in both results. Quantitatively, our DL model obtained significantly smaller PDE residual and boundary condition residual on both the training and testing datasets than the FEM $(p<0.002$, Wilcoxon signed-rank test) as demonstrated in Fig. $5 b$ and Fig. $5 c$, indicating that the results by our DL model are better fit to the governing equations of TMS induced E-fields. Moreover, our DL model obtained comparable residuals on both training and testing subjects, demonstrating the model's robustness to the anatomical differences across different subjects. 

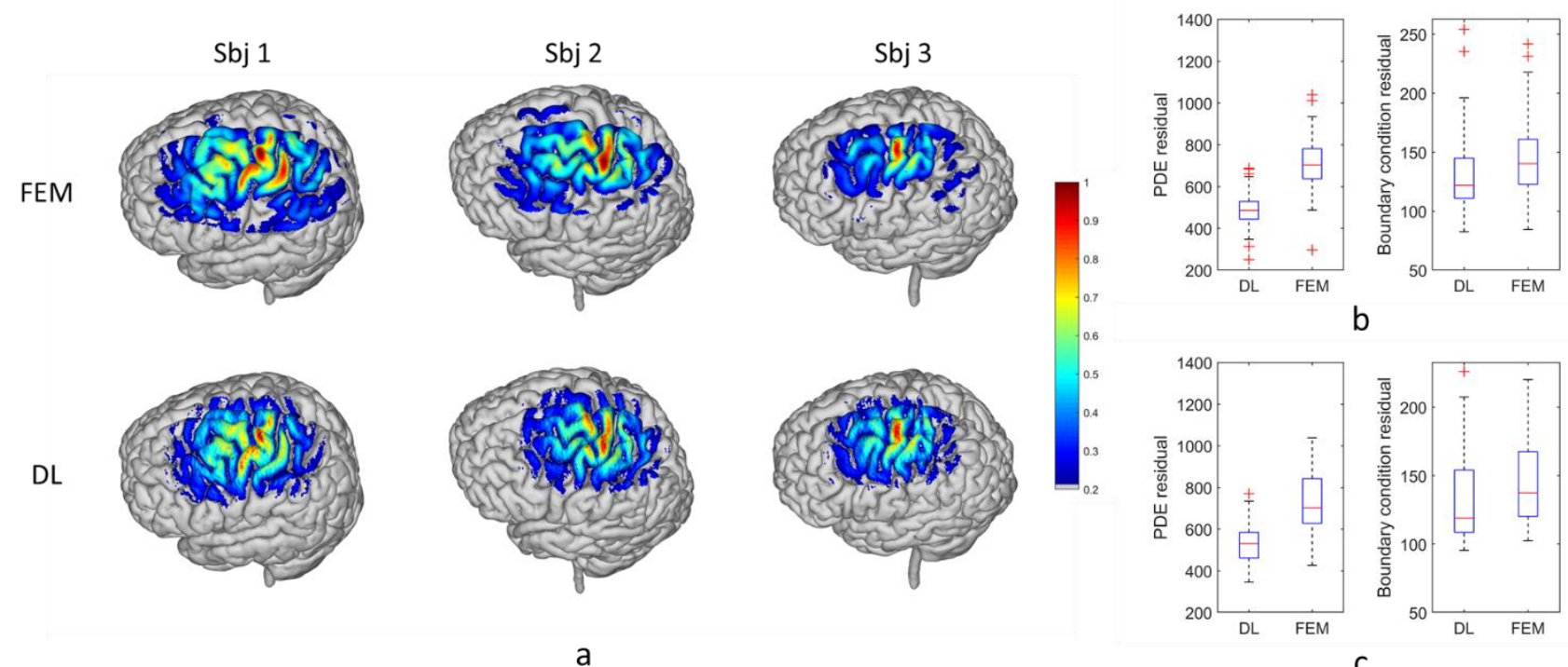

b
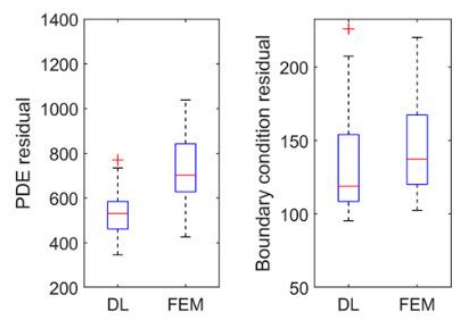

C

Fig.5. E-fields computed by FEM and the proposed DL model for the motor cortex. (a) The magnitude of E-fields of three randomly selected testing subjects computed by FEM and the DL model respectively, at a threshold of 0.2. (b and c) Our DL model performed better than the FEM on both training (b) and testing (c) datasets with respect to the PDE residual and boundary condition residual ( $p<0.002$, Wilcoxon signed-rank test).

Results on realistic head model setting 2. The magnitude of E-fields of three randomly selected testing subjects with unseen coil directions computed by the FEM and our proposed DL method are shown in Fig. 6a. Though the coil directions different from those used for training the DL model, the DL model still successfully predicted the E-fields for the testing subjects, demonstrating good consistency with those obtained by the FEM. Quantitatively, our DL model obtained significantly smaller PDE residual on both seen and unseen coil direction compared with FEM ( $p<0.001$, Wilcoxon signed-rank test) as demonstrated in Fig. 6b and Fig. 6c, with smaller or comparable boundary condition residual $(p<0.001$ for seen coil directions and $p=0.314$ for unseen coil directions, Wilcoxon signed-rank test). It is worth noting that all these results were obtained for testing subjects which were isolated from the model training. These results demonstrated that the DL model was robust to inter-subject anatomical differences under different coil direction settings and generalized well to unseen coil directions. 
Sbj 1

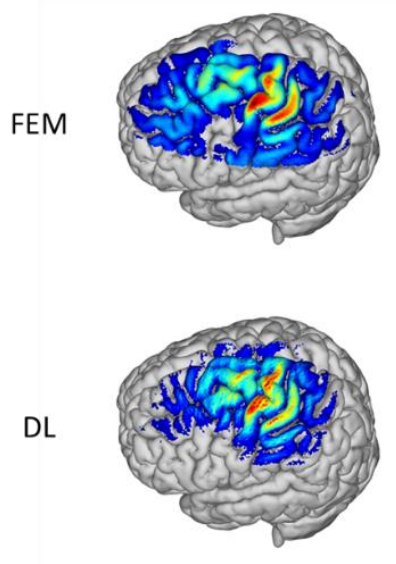

Sbj 2
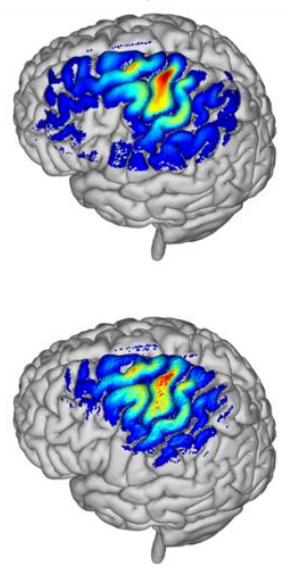

a
Sbj 3
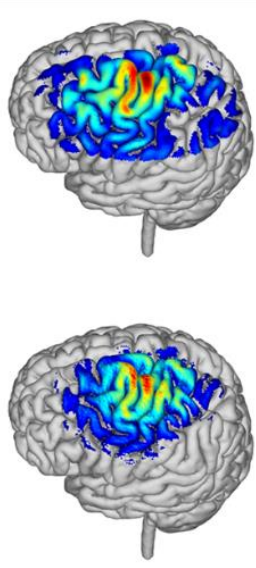
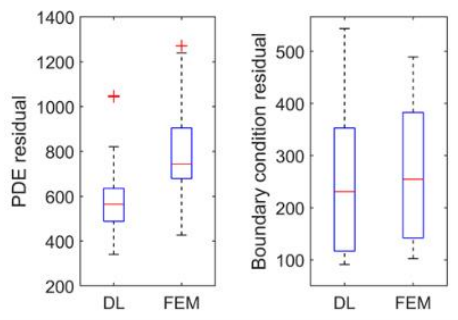

b
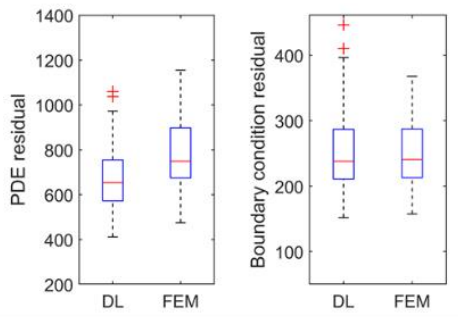

Fig.6. E-fields computed by FEM and the proposed DL model for the motor cortex with varying coil directions. (a) The magnitude of E-fields of three randomly selected testing subjects with unseen coil directions computed by FEM and the DL model respectively, at a threshold of 0.2. (b) Our DL model performed better than the FEM for seen coil directions on testing datasets with respect to both the PDE residual and boundary condition residual $(p<0.001$, Wilcoxon signed-rank test). (c) Our DL model performed better than the FEM for unseen coil directions on testing datasets with respect to the PDE residual $(p<0.001)$, with comparable boundary condition residual $(p=0.314$, Wilcoxon signed-rank test).

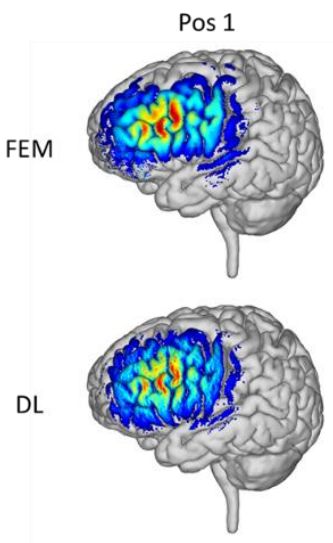

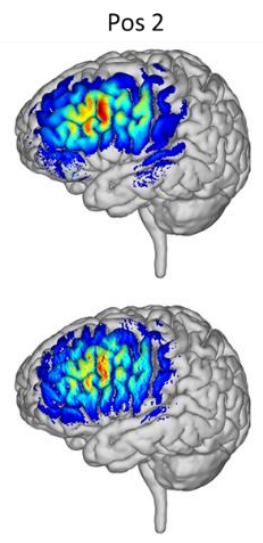

a
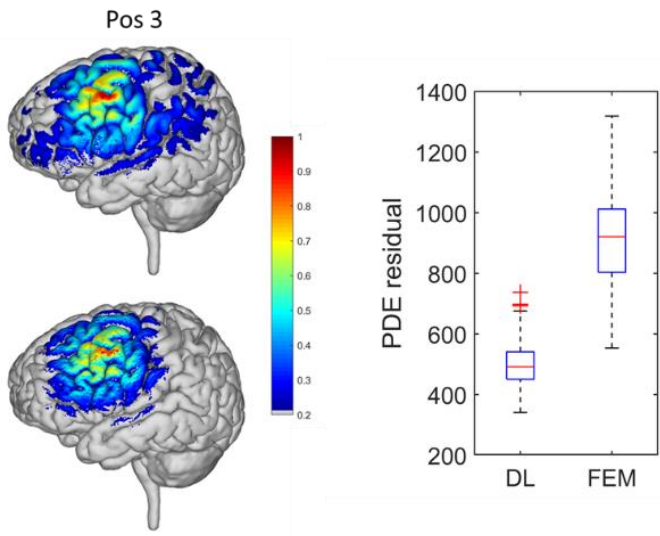

b

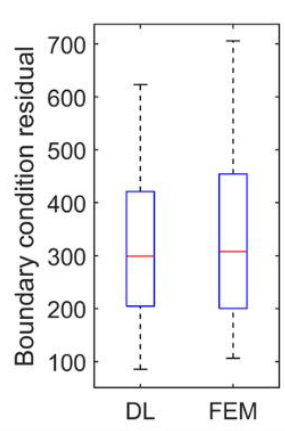

Fig.7. E-fields computed by FEM and the proposed DL model for the dorsolateral prefrontal cortex (DLPFC) with varying coil positions and directions. (a) The magnitude of E-fields of one randomly selected testing subject with three different coil positions computed by FEM and the DL model respectively, at a threshold of 0.2. (b) Our DL model performed better than the FEM on testing datasets with respect to the PDE residual and boundary condition residual ( $p<0.001$, Wilcoxon signed-rank test). 
Results on realistic head model setting 3. The magnitude of E-fields of one randomly selected testing subject computed by FEM and our proposed DL method for three coil positions located within the DLPFC area are shown in Fig. 7a, and the results obtained by our DL model are visually similar to that obtained by FEM. The PDE residuals and boundary condition residuals obtained by our DL model for the testing subjects were significantly smaller than that obtained by FEM ( $p<0.001$, Wilcoxon signed-rank test) as demonstrated in Fig. 7b and Fig. 7c, indicating that our DL model was capable of predicting E-fields with varying coil positions and directions.

Computation time. It took 0.235 and 0.303 seconds on average by our deep learning model to predict the E-field for one testing subject with the TMS coil located at the motor cortex and DLPFC respectively, using one NVIDIA Tesla P100 GPU. As comparison, it took 23.79 and 26.76 seconds on average by FEM as implemented in SIMNIBS, using one Intel Xeon E5-2630 CPU. This comparison demonstrated the improved computational efficiency obtained by the proposed deep learning model.

\section{Discussion}

We have developed a self-supervised deep learning model to directly learn a mapping from the magnetic vector potential of a TMS coil and a realistic head model to the TMS induced E-fields, instead of iteratively solving equations governing the E-field induced by a TMS coil. Experimental results on both simulated and realistic head models have demonstrated that our method could obtain similar or improved accuracy compared with the most commonly used numerical method. Our method is also computationally efficient and capable of computing the TMS induced E-field by one forward-pass computation taking mere milliseconds.

Several numerical computational methods have been developed for accurate E-fields modeling in conjunction with realistic head models, such as FEMs, BEMs, and FDMs. Though the computational efficiency of state-of-the-art numerical methods has been improved a lot, their computational cost for high-resolution E-fields is still high due to the nature of iterative optimization in their PDE solvers, which compromises their use in the optimization of TMS stimulation parameters for both basic and clinical studies. Instead of solving the governing PDEs from scratch, recently studies [32, 33] have demonstrated promising performance of deep neural networks for rapid estimation of E-fields, in which deep neural networks were trained to directly predict the E-fields with high fidelity to E-fields estimated using conventional E-field modeling methods, such as FEMs. Therefore, the deep neural networks are actually trained to predict the solutions obtained by the conventional E-field modeling methods and their performance are bounded by the conventional E-field modeling methods used for generating training data. Moreover, it will also be time-consuming to generate surrogate training data with different TMS 
stimulation parameters on a large cohort.

In contrast to the existing deep learning based E-field modeling methods [32, 33] that learn a mapping from head scans/models to surrogate E-fields estimated using conventional E-field modeling methods in the supervised learning way, our method directly learns a solution to the governing equations with boundary conditions in a self-supervised learning way, which does not require any external supervision. Our proposed deep neural network is designed to predict the TMS induced electric scalar potential and is optimized so that the network's output fit the governing PDE and Neumann boundary condition as much as possible, which is formulated to minimize the residuals of the governing PDE and boundary condition. Therefore, our method directly learns a solution to the same governing PDE and boundary condition as the convention numerical optimization methods do, while benefiting from the fast inference of deep neural networks. As surrogate E-fields are not required by our method, realistic head models from diverse imaging datasets can be used as the training data for our method. To the best of our knowledge, our method is the first study to investigate self-supervised deep learning for TMS E-field modeling, though physics-informed deep learning methods have been successfully applied to solving PDEs on varied domains [34-42].

In addition to improved accuracy and computational efficiency, our method is robust to intersubject anatomical differences and varying TMS coil locations and directions. As demonstrated in Fig. 6, the deep neural network generalized well for computing E-fields of the testing subjects that were generated by the coil placed in directions different from those for generating the training data. The validation experiment with the coil placed at left DLPFC has further demonstrated that the accuracy of the predicted E-fields still outperformed that obtained by the FEM on the testing subjects, even though the coil was placed at varying locations and in different directions, as illustrated in Fig. 7. The good generalization performance of the proposed method may be attributed to its self-supervised learning nature, which optimizes the deep learning model to learn the underlying mapping between head anatomy and electric potential without external guidance or prior assumptions. The robust generalization emphasizes that this method is likely to be applicable to the optimization of TMS stimulation where varying positions and directions around the target position are to be explored.

Though the self-supervised deep learning method has demonstrated great potential for fast and accurate E-field modeling, there are still several limitations should be noted. First, current evaluation was focused on E-field induced by a Figure-of-Eight coil, the influence of different types of coil merits further investigation. Second, scalar tissue conductivity maps were used currently, improved performance is expected when anisotropic tissue conductivity maps are used as input to the deep neural network. Third, our current model adopts a traditional U-Net architecture, which can be optimized in terms of both accuracy and efficiency of the E-field modeling using neural architecture search (NAS) techniques [54]. 
In conclusion, a self-supervised deep learning model was developed to estimate TMS induced Efields directly from realistic head models and the TMS coil's magnetic vector potential. The DL model can obtain high-resolution E-fields from realistic head models with high accuracy within milliseconds, facilitating real-time and precise TMS E-field modeling, and therefore the optimization of TMS stimulation parameters in both basic and clinical studies.

\section{Declarations of interest}

None.

\section{Acknowledgements}

This work was supported in part by the National Institutes of Health [grant numbers: MH120811, EB022573, and AG066650].

\section{References}

[1] O'Reardon JP, Solvason HB, Janicak PG, Sampson S, Isenberg KE, Nahas Z, et al. Efficacy and Safety of Transcranial Magnetic Stimulation in the Acute Treatment of Major Depression: A Multisite Randomized Controlled Trial. Biological Psychiatry 2007;62(11):1208-16.

[2] Fox MD, Buckner RL, White MP, Greicius MD, Pascual-Leone A. Efficacy of transcranial magnetic stimulation targets for depression is related to intrinsic functional connectivity with the subgenual cingulate. Biol Psychiatry 2012;72(7):595-603.

[3] Fox MD, Liu H, Pascual-Leone A. Identification of reproducible individualized targets for treatment of depression with TMS based on intrinsic connectivity. Neurolmage 2013;66:151-60.

[4] Opitz A, Fox MD, Craddock RC, Colcombe S, Milham MP. An integrated framework for targeting functional networks via transcranial magnetic stimulation. Neurolmage 2016;127:86-96.

[5] Weigand A, Horn A, Caballero R, Cooke D, Stern AP, Taylor SF, et al. Prospective Validation That Subgenual Connectivity Predicts Antidepressant Efficacy of Transcranial Magnetic Stimulation Sites. Biol Psychiatry 2018;84(1):28-37.

[6] Diekhoff-Krebs S, Pool EM, Sarfeld AS, Rehme AK, Eickhoff SB, Fink GR, et al. Interindividual differences in motor network connectivity and behavioral response to iTBS in stroke patients. Neurolmage Clinical 2017;15:559-71.

[7] Kim JH, Kim DW, Chang WH, Kim YH, Kim K, Im CH. Inconsistent outcomes of transcranial direct current stimulation may originate from anatomical differences among individuals: electric field simulation using individual MRI data. Neuroscience letters 2014;564:6-10.

[8] Luber BM, Davis S, Bernhardt E, Neacsiu A, Kwapil L, Lisanby SH, et al. Using neuroimaging to individualize TMS treatment for depression: Toward a new paradigm for imaging-guided intervention. Neurolmage 2017;148:1-7. 
[9] Cash RFH, Cocchi L, Lv J, Wu Y, Fitzgerald PB, Zalesky A. Personalized connectivity-guided DLPFC-TMS for depression: Advancing computational feasibility, precision and reproducibility. Human Brain Mapping 2021.

[10] Cash RFH, Weigand A, Zalesky A, Siddiqi SH, Downar J, Fitzgerald PB, et al. Using Brain Imaging to Improve Spatial Targeting of Transcranial Magnetic Stimulation for Depression. Biological Psychiatry 2020.

[11] Williams LM, Coman JT, Stetz PC, Walker NC, Kozel FA, George MS, et al. Identifying response and predictive biomarkers for Transcranial magnetic stimulation outcomes: protocol and rationale for a mechanistic study of functional neuroimaging and behavioral biomarkers in veterans with Pharmacoresistant depression. BMC Psychiatry 2021;21(1).

[12] Sack AT, Kadosh RC, Schuhmann T, Moerel M, Walsh V, Goebel R. Optimizing Functional Accuracy of TMS in Cognitive Studies: A Comparison of Methods. Journal of Cognitive Neuroscience 2009;21(2):207-21.

[13] Julkunen P, Saisanen L, Danner N, Niskanen E, Hukkanen T, Mervaala E, et al. Comparison of navigated and non-navigated transcranial magnetic stimulation for motor cortex mapping, motor threshold and motor evoked potentials. Neurolmage 2009;44(3):790-5.

[14] Weiss C, Nettekoven C, Rehme AK, Neuschmelting V, Eisenbeis A, Goldbrunner R, et al. Mapping the hand, foot and face representations in the primary motor cortex - retest reliability of neuronavigated TMS versus functional MRI. Neurolmage 2013;66:531-42.

[15] Weise K, Numssen O, Thielscher A, Hartwigsen G, Knosche TR. A novel approach to localize cortical TMS effects. Neurolmage 2020;209:116486.

[16] Makarov SN, Wartman WA, Daneshzand M, Nummenmaa A. A Note about the Individualized TMS Focality. bioRxiv 2020:2020.02.10.941062.

[17] Gomez LJ, Dannhauer M, Peterchev AV. Fast computational optimization of TMS coil placement for individualized electric field targeting. Neurolmage 2021;228:117696.

[18] Deng ZD, Lisanby SH, Peterchev AV. Electric field depth-focality tradeoff in transcranial magnetic stimulation: simulation comparison of 50 coil designs. Brain Stimul 2013;6(1):1-13.

[19] Bungert A, Antunes A, Espenhahn S, Thielscher A. Where does TMS Stimulate the Motor Cortex? Combining Electrophysiological Measurements and Realistic Field Estimates to Reveal the Affected Cortex Position. Cereb Cortex 2017;27(11):5083-94.

[20] Goetz SM, Deng ZD. The development and modelling of devices and paradigms for transcranial magnetic stimulation. Int Rev Psychiatry 2017;29(2):115-45.

[21] Saturnino GB, Madsen $\mathrm{KH}$, Thielscher A. Electric field simulations for transcranial brain stimulation using FEM: an efficient implementation and error analysis. Journal of Neural Engineering 2019;16(6):066032.

[22] Wang W, Eisenberg SR. A three-dimensional finite element method for computing magnetically induced currents in tissues. IEEE Transactions on Magnetics 1994;30(6):5015-23.

[23] Gomez-Tames J, Laakso I, Hirata A. Review on biophysical modelling and simulation studies for transcranial magnetic stimulation. Physics in Medicine \& Biology 2020;65(24). 
[24] Makarov SN, Wartman WA, Daneshzand M, Fujimoto K, Raij T, Nummenmaa A. A software toolkit for TMS electric-field modeling with boundary element fast multipole method: an efficient MATLAB implementation. Journal of Neural Engineering 2020;17(4).

[25] Paffi A, Camera F, Carducci F, Rubino G, Tampieri P, Liberti M, et al. A Computational Model for Real-Time Calculation of Electric Field due to Transcranial Magnetic Stimulation in Clinics. International Journal of Antennas and Propagation 2015;2015:1-11.

[26] Nielsen JD, Madsen KH, Puonti O, Siebner HR, Bauer C, Madsen CG, et al. Automatic skull segmentation from MR images for realistic volume conductor models of the head: Assessment of the state-of-the-art. Neurolmage 2018;174:587-98.

[27] Htet AT, Saturnino GB, Burnham EH, Noetscher GM, Nummenmaa A, Makarov SN. Comparative performance of the finite element method and the boundary element fast multipole method for problems mimicking transcranial magnetic stimulation (TMS). Journal of Neural Engineering 2019;16(2):024001.

[28] Gomez LJ, Dannhauer M, Koponen LM, Peterchev AV. Conditions for numerically accurate TMS electric field simulation. Brain Stimul 2020;13(1):157-66.

[29] Babuska I, Szabo BA, Katz IN. The p-Version of the Finite Element Method. SIAM Journal on Numerical Analysis 1981;18(3):515-45.

[30] Daneshzand M, Makarov SN, de Lara LIN, Guerin B, McNab J, Rosen BR, et al. Rapid computation of TMSinduced E-fields using a dipole-based magnetic stimulation profile approach. Neurolmage 2021;237:118097.

[31] Koponen LM, Stenroos M, Nieminen JO, Jokivarsi K, Gröhn O, Ilmoniemi RJ. Individual head models for estimating the TMS-induced electric field in rat brain. bioRxiv 2019.

[32] Yokota T, Maki T, Nagata T, Murakami T, Ugawa Y, Laakso I, et al. Real-time estimation of electric fields induced by transcranial magnetic stimulation with deep neural networks. Brain Stimul 2019;12(6):1500-7.

[33] Xu G, Rathi Y, Camprodon JA, Cao H, Ning L. Rapid whole-brain electric field mapping in transcranial magnetic stimulation using deep learning. PLoS One 2021;16(7):e0254588.

[34] Raissi M, Perdikaris P, Karniadakis GE. Physics-informed neural networks: A deep learning framework for solving forward and inverse problems involving nonlinear partial differential equations. Journal of Computational Physics 2019;378:686-707.

[35] Qin T, Wu K, Xiu D. Data driven governing equations approximation using deep neural networks. Journal of Computational Physics 2019;395:620-35.

[36] Tian F-B, Kadeethum T, Jørgensen TM, Nick HM. Physics-informed neural networks for solving nonlinear diffusivity and Biot's equations. Plos One 2020;15(5).

[37] Guo Y, Cao X, Liu B, Gao M. Solving Partial Differential Equations Using Deep Learning and Physical Constraints. Applied Sciences 2020;10(17).

[38] Geneva N, Zabaras N. Modeling the dynamics of PDE systems with physics-constrained deep auto-regressive networks. Journal of Computational Physics 2020;403. 
[39] Zhu Y, Zabaras N, Koutsourelakis P-S, Perdikaris P. Physics-constrained deep learning for high-dimensional surrogate modeling and uncertainty quantification without labeled data. Journal of Computational Physics 2019;394:56-81.

[40] Yang Y, Perdikaris P. Adversarial uncertainty quantification in physics-informed neural networks. Journal of Computational Physics 2019;394:136-52.

[41] Winovich N, Ramani K, Lin G. ConvPDE-UQ: Convolutional neural networks with quantified uncertainty for heterogeneous elliptic partial differential equations on varied domains. Journal of Computational Physics 2019;394:263-79.

[42] Rao C, Sun H, Liu Y. Physics informed deep learning for computational elastodynamics without labeled data. arXiv preprint arXiv:200608472 2020.

[43] Li H, Dhivya S, Cui Z, Zhuo C, Gur RE, Gur RC, et al. Computing personalized brain functional networks from fMRI using self-supervised deep learning. bioRxiv 2021:2021.09.25.461829.

[44] Li H, Fan Y. Non-rigid image registration using self-supervised fully convolutional networks without training data. 2018 IEEE 15th International Symposium on Biomedical Imaging (ISBI 2018). 2018:1075-8.

[45] Li H, Fan Y. MDReg-Net: Multi-resolution diffeomorphic image registration using fully convolutional networks with deep self-supervision. arXiv: 2010014652020.

[46] Koponen LM, Nieminen JO, Mutanen TP, Stenroos M, Ilmoniemi RJ. Coil optimisation for transcranial magnetic stimulation in realistic head geometry. Brain Stimul 2017;10(4):795-805.

[47] Ronneberger O, Fischer P, Brox T. U-net: Convolutional networks for biomedical image segmentation. International Conference on Medical image computing and computer-assisted intervention. Springer; 2015:234-41.

[48] Maas AL, Hannun AY, Ng AY. Rectifier nonlinearities improve neural network acoustic models. Proc. icml. 30. Citeseer; 2013:3.

[49] Heller L, van Hulsteyn DB. Brain stimulation using electromagnetic sources: theoretical aspects. Biophysical journal 1992;63(1):129-38.

[50] Gomez-Tames J, Hamasaka A, Laakso I, Hirata A, Ugawa Y. Atlas of optimal coil orientation and position for TMS: A computational study. Brain Stimul 2018;11(4):839-48.

[51] Friehs MA, Klaus J, Singh T, Frings C, Hartwigsen G. Perturbation of the right prefrontal cortex disrupts interference control. Neurolmage 2020;222:117279.

[52] Abadi M, Barham P, Chen J, Chen Z, Davis A, Dean J, et al. Tensorflow: A system for large-scale machine learning. 12th \{USENIX\} symposium on operating systems design and implementation (\{OSDI\} 16). 2016:26583.

[53] Kingma DP, Ba J. Adam: A method for stochastic optimization. arXiv preprint arXiv:14126980 2014.

[54] Elsken T, Metzen JH, Hutter F. Neural architecture search: A survey. The Journal of Machine Learning Research 2019;20(1):1997-2017. 